\title{
Estudo descritivo sobre a importância da avaliação funcional como procedimento prévio no controle fisiológico do treinamento físico de futebolistas realizado em pré-temporada
}

\author{
Alberto Azevedo Alves Teixeira ${ }^{1}$, Paulo Roberto Santos Silva ${ }^{2}$, Luís Antonio Inarra ${ }^{3}$, \\ José Roberto Rivelino Vidal ${ }^{3}$, Cláudio Lépera ${ }^{4}$, Gilberto Silva Machado ${ }^{5}$, \\ Luciana Collet Winther Rebello ${ }^{6}$, Luís Carlos Prima ${ }^{7}$, Mário Jorge Lobo Zagallo ${ }^{8}$ \\ e Jorge Mendes de Sousa ${ }^{9}$
}

Seção de Fisiologia do Centro de Medicina Integrada e Seção de Preparação Física do Departamento de Futebol Profissional da Associação Portuguesa de Desportos de São Paulo

\section{RESUMO}

O principal objetivo deste estudo foi mostrar a importância da avaliação funcional como procedimento utilizado no controle fisiológico do treinamento físico de futebolistas profissionais em pré-temporada. Foram avaliados e posteriormente concentrados na cidade de Águas de Lindóia, Estado de São Paulo, por 16 dias, 23 jogadores pertencentes ao Departamento de Futebol Profissional da Associação Portuguesa de Desportos, em preparação para o Campeonato Paulista, edição 1999. Todos foram submetidos a uma bateria de testes que constou de avaliação: cardiorrespiratória e metabólica, odontológica, isocinética de membros inferiores, percentagem de gordura corpórea, da potência anaeróbia pelo teste de Wingate e testes de campo. Os seguintes resultados e os parâmetros avaliados foram: no limiar ventilatório $2\left(\mathbf{L V}_{2}\right): \mathrm{VO}_{2}=49,09 \pm 4,83 \mathrm{ml}$. $\mathrm{kg}^{-1} \cdot \mathrm{min}^{-1} ; \% \mathrm{VO}_{2}=82,7 \pm 5,8 ;$ velocidade de corrida $=12,8 \pm$ $0,9 \mathrm{~km} \cdot \mathrm{h}^{-1} ; \mathrm{FC}=174 \pm 9 \mathrm{bpm}$; no exercício máximo: $\mathrm{V}_{\mathrm{E} \text { втРS }}$ $=137,3 \pm 11,3 \mathrm{~L} \cdot \mathrm{min}^{-1}$; velocidade de corrida $=17,6 \pm 0,7 \mathrm{~km}$.

1. Médico do Esporte.

2. Fisiologista.

3. Fisicultor.

4. Ortopedista.

5. Fisioterapeuta.

6. Nutricionista.

7. Auxiliar Técnico de Futebol.

8. Técnico de Futebol.

9. Vice-Presidente de Medicina da Associação Portuguesa de DesportosSP.

Endereço para correspondência:

Alberto Azevedo Alves Teixeira

Centro de Medicina Integrada - Seção de Fisiologia

Rua Comendador Nestor Pereira, 33

03034-070 - São Paulo, SP - Brasil

Tel.: (011) 225-0400, ramais 224/228

Fax: (011) 228-2001 $\mathrm{h}^{-1} ; \mathrm{FC}=(191 \pm 8 \mathrm{bpm}) ; \mathrm{VO}_{2}$ pico $=59,28 \pm 3,52 \mathrm{ml} \cdot \mathrm{kg}^{-1}$. $\min ^{-1}$; lactato $=10,5 \pm 1,5 \mathrm{mM}$. Teste de Wingate: potência pico $=13,5 \pm 1,1 \mathrm{w} \cdot \mathrm{kg}^{-1}$; potência média $=10,1 \pm 0,6 \mathrm{w} \cdot \mathrm{kg}^{-1}$; índice de fadiga $=53,0 \pm 7,7 \%$. Teste isocinético: velocidade angular da articulação do joelho da perna direita na extensão e flexão a $60^{\circ} \mathrm{S}^{-1}=298 \pm 72$ e $198 \pm 44 \mathrm{Nm}$, respectivamente; a $300^{\circ} \mathrm{S}^{-1}$ extensão e flexão $=137 \pm 32$ e $121 \pm 26 \mathrm{Nm}$, respectivamente. Velocidade angular da articulação do joelho da perna esquerda na extensão e flexão a $60^{\circ} \mathrm{S}^{-1}=272 \pm 62$ e 185 $\pm 45 \mathrm{Nm}$, respectivamente; a $300^{\circ} \mathrm{S}^{-1}$ extensão e flexão $=138$ \pm 28 e $122 \pm 27 \mathrm{Nm}$, respectivamente. A intensidade dos treinamentos aeróbio e anaeróbio foi controlada em campo por medidas de lactato, utilizando-se lactômetro portátil. O treinamento de musculação foi realizado a $60 \%$ da carga máxima para cada exercício. Os exercícios de alongamento e na piscina (hidroginástica) foram exaustivamente realizados pelos jogadores. Em média, cada sessão (manhã e tarde) durou 120 minutos. Portanto, pelo pouco tempo destinado a essa fase de preparação, a importância da avaliação funcional multidisciplinar justifica-se ainda mais, pois, a partir dos resultados, é possível detectar e corrigir possíveis deficiências, direcionando com objetividade o treinamento dos futebolistas.

Palavras-chave: Avaliação fisiológica. Treinamento físico. Pré-temporada. Jogadores de futebol. Medicina Esportiva.

\section{ABSTRACT \\ Descriptive study of the importance of functional evaluation as a previous procedure for physiological control of physi- cal training in soccer players carried out in a pre-season period}

The main purpose of this study was to show the importance of functional evaluation as a procedure used in the physiological control of pre-season physical training for professional 
soccer players. In a preliminary round aiming at the 1999 São Paulo soccer championship, 23 soccer players belonging to the Departament of Professional Soccer of Associação Portuguesa de Desportos were evaluated and then assembled in Aguas de Lindoia, a resort in São Paulo State, for a 16-day period. All of them were submitted a battery of tests comprising cardiorespiratory, metabolic, odontological, and lower limb isokinetic evaluation, as well as body fat and anaerobic power percentage by the Wingate test and field tests. These were the results and parameters evaluated: in the ventilatory threshold, $2\left(V_{T_{2}}\right): \mathrm{VO}_{2}=49.09 \pm 4.83 \mathrm{ml} \cdot \mathrm{kg}^{-1} \cdot \mathrm{min}^{-1} ; \% \mathrm{VO}_{2}$ $=82.7 \pm 5.8$; running speed $=12.8 \pm 0.9 \mathrm{~km} \cdot \mathrm{h}^{-1} ; \mathrm{HR}=174 \pm$ $9 \mathrm{bpm}$; in maximum exercise: $V_{E \text { BTPS }}=137.3 \pm 11.3 \mathrm{~L} . \mathrm{min}^{-1}$; running speed $=17.6 \pm 0.7 \mathrm{~km} \cdot \mathrm{h}^{-1} ; \mathrm{HR}=191 \pm 8 \mathrm{bpm} ; \mathrm{VO}_{2}$ peak $=59.28 \pm 3.52 \mathrm{ml} \cdot \mathrm{kg}^{-1} \cdot \mathrm{min}^{-1}$; lactic acid $=10.5 \pm 1.5$

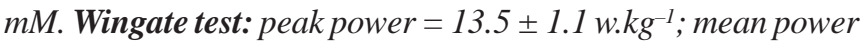
$=10.1 \pm 0.6 \mathrm{w} . \mathrm{kg}^{-1}$; fatigue index $=53.0 \pm 7.7 \%$; isokinetic test: angular speed at $60^{\circ} \mathrm{S}^{-1}$ right leg extension and flexion = $298 \pm 72$ and $198 \pm 44 \mathrm{Nm}$, respectively; at $300^{\circ} \mathrm{S}^{-1}$ extension and flexion $=137 \pm 32$ and $121 \pm 26 \mathrm{Nm}$, respctively. Angular speed at $60^{\circ} \mathrm{S}^{-1}$ left leg extension and flexion $=272 \pm$ 62 and $185 \pm 45 \mathrm{Nm}$, respectively; at $300^{\circ} \mathrm{S}^{-1}$ extension and flexion $138 \pm 28$ and $122 \pm 27 \mathrm{Nm}$, respectively. The intensity of aerobic and anaerobic training was controlled in the field by measuring lactic acid. To this end, the authors used a portable measuring device. The body-building training was carried out at a $60 \%$ maximum charge for each exercise. The limber-up and swimming-pool exercises were exhaustively carried out by the soccer players. Each training session (morning and afternoon) lasted 120 minutes on average. Therefore, considering the short time designed for this phase of preparation, the importance of a multidisciplinary functional evaluation is even more justifiable, for, according to the results, it allows one to detect and correct possible deficiencies with an objectively-oriented soccer player training.

Key words: Physiological evaluation. Physical training. Pre-season. Soccer players. Sports Medicine.

\section{INTRODUÇÃO}

O treinamento físico moderno, realizado com qualidade, deve ser constantemente monitorado por meio de controle fisiológico, direcionado para o desenvolvimento apropriado das qualidades físicas pertinentes à atividade esportiva ${ }^{1-3}$.

A chamada pré-temporada de nossas agremiações é a fase de preparação inicial do ano para as principais competições futebolísticas realizadas no Brasil.

No Estado de São Paulo, a preparação atlética dos jogadores de futebol das principais equipes é realizada em poucas semanas, tempo considerado exíguo.

Logo, a divisão adequada dos treinamentos para o aprimoramento físico, técnico e tático das equipes não é uma tarefa fácil de ser executada pelos especialistas.
Justifica-se, portanto, cada vez mais, a valorização do processo de avaliação funcional do atleta por meio de testes realizados em laboratório de fisiologia do exercício e campo, pois só assim é possível verificar de maneira precisa as necessidades e corrigir as deficiências encontradas nos jogadores de futebol ${ }^{4}$.

É importante salientar que o planejamento de construção, evolução e adequação das cargas de treinamento depende essencialmente dos resultados individuais de cada atleta. Portanto, desse modo, é possível desenvolver adaptações funcionais mínimas compatíveis com a prática do futebol.

O principal objetivo deste estudo foi descrever procedimentos de controle fisiológico do treinamento físico em futebolistas, proveniente de testes realizados em laboratório de fisiologia do exercício, complementados por testes de campo, como requisito para o desenvolvimento adequado da preparação física dos jogadores de futebol em pré-temporada futebolística.

\section{MATERIAL E MÉTODOS}

Foram concentrados para a pré-temporada futebolística na cidade de Águas de Lindóia, uma instância turística no Estado de São Paulo, 23 jogadores de futebol profissional, com idade variando de 21 a 33 anos (tabela 1). Todos os futebolistas eram pertencentes ao Departamento de Futebol Profissional da Associação Portuguesa de Desportos, São Paulo-SP, em preparação para o Campeonato Paulista de Futebol, edição 1999.

Os atletas foram submetidos à avaliação eletrocardiográfica em repouso e durante o teste de esforço por meio da monitoração de 12 derivações simultâneas segundo disposição preconizada por Mason \& Likar, com modificação da derivação $\mathrm{D}_{1}$ para $\mathrm{MC}_{5}$, utilizando-se eletrocardiógrafo computadorizado (modelo 6.4, Heart-Ware, BRA). A pressão arterial (PA) foi medida por método auscultatório indireto, utilizando-se esfigmomanômetro aneróide (Tycos, USA).

A ventilação pulmonar $\left(\mathrm{V}_{\mathrm{E} \text { BTPS }}\right)$, o consumo de oxigênio $\left(\mathrm{VO}_{2 \text { STPD }}\right)$, a produção de dióxido de carbono $\left(\mathrm{VCO}_{2 \text { STPD }}\right)$ e a razão de troca respiratória $\left(\mathrm{RER}=\mathrm{VCO}_{2} / \mathrm{VO}_{2}\right)$ foram calculados por meio de valores medidos por um sistema computadorizado de análise de troca gasosa (respiração-a-respiração Breath-by-Breath [B x B]) (modelo Vmax 29c, SensorMedics,

\section{TABELA 1}

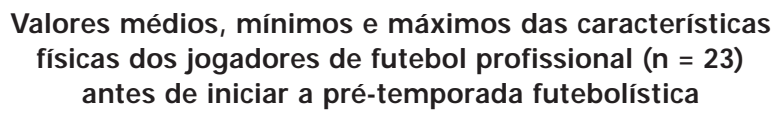

\begin{tabular}{cccc}
\hline $\begin{array}{c}\text { Idade } \\
\text { (anos) }\end{array}$ & $\begin{array}{c}\text { Peso } \\
(\mathbf{k g})\end{array}$ & $\begin{array}{c}\text { Estatura } \\
(\mathbf{c m})\end{array}$ & $\begin{array}{c}\text { Gordura } \\
\text { (\%) }\end{array}$ \\
24,8 & 72,8 & 178,5 & 9,8 \\
$\pm 3,3$ & $\pm 6,4$ & $\pm 5,3$ & $\pm 0,9$ \\
$(21-33)$ & $(57-100)$ & $(160-189)$ & $(8,2-12,8)$ \\
\hline
\end{tabular}


USA). O volume ventilatório foi medido por um sensor de fluxo de massa (SensorMedics, USA).

A calibração do sensor de fluxo de massa foi feita antes da realização de cada teste, com uma seringa de 3 litros, para ser empregado fator de correção que determinará o volume respiratório. As frações expiradas de oxigênio $\left(\mathrm{F}_{\mathrm{E}} \mathrm{O}_{2}\right)$ foram medidas por um sensor paramagnético de resposta rápida e elevada precisão (SensorMedics, USA) e as frações expiradas de dióxido de carbono $\left(\mathrm{F}_{\mathrm{E}} \mathrm{CO}_{2}\right)$ pelo princípio infravermelho.

A calibração dos analisadores do equipamento foi feita antes e imediatamente após a realização de cada teste, com mistura gasosa conhecida de $\mathrm{O}_{2}(12 \%$ e $26 \%), \mathrm{CO}_{2}(4 \%)$ e balanceada com nitrogênio $\left(\mathrm{N}_{2}\right)$. As variáveis ventilatórias foram registradas instantaneamente e depois calculadas para o tempo médio de 10 segundos.

A avaliação da capacidade funcional máxima dos futebolistas foi verificada realizando-se um teste de esforço em esteira rolante (modelo ATL-10.200, Inbramed, BRA) de velocidade $\left(\mathrm{km} . \mathrm{h}^{-1}\right)$ e inclinação $(\%)$ variáveis, utilizando-se protocolo escalonado contínuo e inclinação fixa de $3 \%$.

Nesse protocolo, o atleta ficou dois minutos em repouso, foi aquecido por quatro minutos nas velocidades 4, 5, $6 \mathrm{e}$ $7 \mathrm{~km} . \mathrm{h}^{-1}$ durante um minuto em cada velocidade. Posteriormente, iniciou-se o teste com $8 \mathrm{~km} . \mathrm{h}^{-1} \mathrm{e}$ incrementos de $2 \mathrm{~km}$. $\mathrm{h}^{-1}$ a cada dois minutos até a exaustão do atleta. A fase de recuperação durou quatro minutos e foi realizada com velocidades controladas a $60 \%, 50 \%$ e $40 \%$ da velocidade máxima atingida pelo atleta no teste. Do terceiro ao quarto minuto de recuperação, o atleta foi sentado para coleta de lactato sanguíneo. A percepção subjetiva ao esforço foi verificada em cada estágio do teste pela escala linear gradual de 15 pontos (6 a 20) de Borg 5 .

A capacidade anaeróbia máxima foi estimada por coleta de lactato sanguíneo da polpa de dedo, no terceiro minuto da fase de recuperação do teste, utilizando-se um lactômetro portátil (modelo Accusport, Boehringer Mannheim, GER).

$\mathrm{O}$ limiar ventilatório $2\left(\mathrm{LV}_{2}\right)$ foi determinado utilizando-se os seguintes critérios: 1) menor valor do equivalente ventilatório de dióxido de carbono $\left(\mathrm{V}_{\mathrm{E}} \cdot \mathrm{VCO}_{2}^{-1}\right)$ seguido de inflexão e incremento ao longo do teste; e 2) maior valor da fração expirada de dióxido de carbono $\left(\mathrm{F}_{\mathrm{E}} \mathrm{CO}_{2}\right)$ seguido de inflexão e diminuição progressiva durante o teste. Ambos os critérios foram verificados em exercício de intensidade progressiva ${ }^{7}$.

As potências anaeróbias alática, lática e o índice de fadiga foram estimadas por método não invasivo, utilizando-se o teste de Wingate. O equipamento utilizado foi uma bicicleta (modelo Bike, Cybex, USA) com um sistema computadorizado de alta precisão. Antes de iniciar o teste, o atleta foi aquecido durante cinco minutos em uma bicicleta eletromagnética (modelo EC-1500, Cataye, USA), com uma carga equivalente a $25 \mathrm{w}$ e pedalando a uma velocidade média de 60rpm. Posteriormente à fase de aquecimento, o teste durou 30 segundos, com o atleta sentado e pedalando na mais alta velocidade pos- sível, com uma carga inicial correspondente a $10 \%(0,10 \mathrm{~kg})$ do seu peso corporal ${ }^{8}$. O teste permitiu estimar a potência anaeróbia alática por meio do pico de potência absoluta (watts) e relativa à superfície corpórea (watts. $\mathrm{kg}^{-1}$ ), atingida aproximadamente entre os três e cinco segundos. A potência anaeróbia lática, ou potência média, foi estimada ao final dos 30 segundos de teste e registrada com as mesmas unidades anteriores citadas. O índice, ou taxa de fadiga percentual, foi calculado por meio da menor potência dividida pela maior potência atingida ao final do teste ${ }^{8}$.

Todos os futebolistas foram submetidos à avaliação odontológica por meio de anamnese e exame clínico, que constaram da verificação de possíveis cáries, restaurações a serem trocadas e indicação de endodontia. Os aparelhos utilizados durante os procedimentos de avaliação foram: espelho clínico e explorador $\mathrm{n}$ 5, ambos em um consultório (modelo MLX Plus, Funk, BRA). Nos casos em que houve dúvidas sobre a existência de cáries interproximais foram utilizadas radiografias oclusais com filmes Kodak no equipamento de raios X (modelo Spectro II, Dabi Atlante, BRA).

A percentagem de gordura corporal foi verificada por meio de medidas de dobras cutâneas, utilizando-se o equipamento plicômetro (Cescorf, BRA). Os pontos anatômicos medidos por três vezes, sempre do lado direito e pelo mesmo avaliador, foram os seguintes:

1) Subescapular: imediatamente abaixo do ângulo inferior da escápula, sendo a dobra cutânea feita obliquamente $\left(45^{\circ}\right)$ ao eixo longitudinal.

2) Tríceps: ponto médio entre o acrômio e a olécrano, na face posterior do braço estendido ao longo do corpo, sendo a dobra cutânea feita na direção do eixo longitudinal.

3) Supra-ilíaca: ponto localizado 3 a $5 \mathrm{~cm}$ do processo ilíaco ântero-superior, sendo a dobra cutânea tomada obliquamente.

4) Abdominal: dobra horizontalmente tomada à cicatriz umbilical.

Foi utilizada a fórmula de Yuhasz, modificada por Faulkner, que determina a percentagem de gordura, por meio da seguinte equação: [\% gordura $=\sum$ das 4 dobras x $0,153+$ $5,783]^{9}$.

Os jogadores de futebol foram submetidos à avaliação músculo-esquelética isocinética computadorizada da articulação dos joelhos no equipamento dinamômetro (modelo 1200, $C y$ bex, USA) nas velocidades angulares de $60^{\circ} \mathrm{e} 300^{\circ}$ por segundo $\left({ }^{\circ} \mathrm{S}^{-1}\right)$. O protocolo de teste foi realizado por meio de cinco movimentos repetitivos na velocidade de 60 e de 30 repetições a $300^{\circ} \mathrm{S}^{-1}$. Antes de iniciar o teste propriamente dito, o atleta foi colocado sentado e iniciou-se um ensaio por meio de duas ou três repetições simuladas em intensidade submáxima para adaptação e reconhecimento de cada velocidade. Posteriormente, o teste iniciou-se com flexão e extensão do joelho, em esforço de intensidade máxima, com um intervalo de 60 segundos entre as velocidades testadas. 
Para verificar a aptidão física em campo foram realizados testes máximos, específicos em várias distâncias (100m, 200m, $300 \mathrm{~m}$ e $1.000 \mathrm{~m}$ ), para posteriormente calcular a percentagem da carga máxima, que foi utilizada em função do treinamento da qualidade física alvo.

Foi utilizada como critério de referência no controle de intensidade do treinamento físico aeróbio contínuo e intervalado a concentração de lactato a ponto fixo $\leq$ a 4,0mM. A velocidade de corrida durante os treinamentos para desenvolvimento da capacidade aeróbia foi calculada a $90 \%$ da velocidade do limiar ventilatório $2\left(\mathrm{LV}_{2}\right)$, verificado no teste ergoespirométrico computadorizado (gases expirados).

Quando foi realizado treinamento físico objetivando a capilarização, o referencial de intensidade do exercício foi controlado por medida de lactato a ponto fixo $\leq 2,0 \mathrm{mM}$.

Os treinamentos físicos, anaeróbios alático e lático, foram realizados a intensidades entre $90 \%$ e $95 \%$ da velocidade máxima para a distância específica e o controle fisiológico efetuado por medidas de lactato sanguíneo.

Os exercícios de musculação foram realizados a uma intensidade correspondente a $60 \%$ da carga máxima atingida no teste.

Nas sessões de treinamento, o alongamento muscular e a mobilidade articular de membros inferiores associado a exer-

\section{TABELA 2}

Valores médios, mínimos e máximos de $\mathrm{VO}_{2}\left(\mathrm{ml} \cdot \mathrm{kg}^{-1} \cdot \mathrm{min}^{-1} \mathrm{e}\right.$ $\left.\% \mathrm{VO}_{\text {2pico }}\right)$, velocidade de corrida $\left(\mathbf{k m} \cdot \mathrm{h}^{-1}\right)$ e freqüência cardíaca (bpm) no limiar ventilatório 2 determinado em teste de esforço incremental em esteira, em jogadores de futebol profissional $(n=23)$, antes do início da pré-temporada

\begin{tabular}{|c|c|c|c|}
\hline 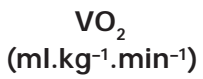 & $\begin{array}{l}\mathrm{VO}_{2} \\
\text { (\%) }\end{array}$ & $\begin{array}{l}\text { Veloc. comida } \\
\left(\mathbf{k m} \cdot \mathbf{h}^{-1}\right)\end{array}$ & $\begin{array}{c}\text { FC } \\
(\text { bpm) }\end{array}$ \\
\hline 49,09 & 82,7 & 13,3 & 174 \\
\hline $\pm 4,83$ & $\pm 5,8$ & $\pm 0,9$ & \pm 9 \\
\hline$(40,8-58,6)$ & (72-95) & (12-14) & (151-189) \\
\hline
\end{tabular}

TABELA 3

Valores médios, mínimos e máximos de $\mathrm{V}_{\mathrm{E}}\left(\right.$ L.min $\left.^{-1}\right)$, velocidade de corrida $\left(\mathrm{km}^{-1}\right)$, freqüência cardíaca (bpm), $\mathrm{VO}_{\text {2pico }}\left(\mathrm{ml} \mathrm{kg}^{-1} \cdot \mathrm{min}^{-1}\right)$ e concentração de lactato $(\mathrm{mM})$, determinado no esforço máximo em teste incremental na esteira, em jogadores de futebol profissional ( $n=23)$, antes do início da pré-temporada

\begin{tabular}{|c|c|c|c|c|}
\hline $\begin{array}{l}V_{\text {E BTPS }} \\
\left(\text { L. } \min ^{-1}\right)\end{array}$ & $\begin{array}{l}\text { Veloc. corrida } \\
\left(\mathbf{k m} \cdot \mathbf{h}^{-1}\right)\end{array}$ & $\begin{array}{c}\text { FC } \\
\text { (bpm) }\end{array}$ & 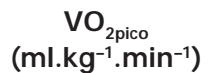 & $\begin{array}{l}\text { Lactato } \\
\text { (mM) }\end{array}$ \\
\hline 137,3 & 17,6 & 191 & 59,28 & 10,5 \\
\hline $\pm 11,3$ & $\pm 0,7$ & \pm 8 & $\pm 3,52$ & $\pm 1,5$ \\
\hline$(118,1-155,7)$ & $(16-18)$ & (176-206) & $(49,3-63,1)$ & $(7,5-13,2)$ \\
\hline
\end{tabular}

cícios na piscina (hidroginástica) foram exaustivamente realizados durante a pré-temporada.

Análise estatística: Os dados foram analisados calculando-se a média, o desvio-padrão e as variações mínima e máxi$\mathrm{ma}^{10}$.

\section{RESULTADOS}

Os resultados estão listados nas tabelas 1, 2, 3, 4, 5, 6 e 7 .

\section{COMENTÁRIOS}

A construção e a manutenção da aptidão funcional de um grupo de atletas em preparação para o ano competitivo deve ser apoiada em uma estrutura multidisciplinar que garanta a eles o desenvolvimento adequado das qualidades físicas para a prática de sua atividade esportiva em elevado nível orgâni$\mathrm{co}^{3}$.

A melhoria da capacidade de um atleta tolerar a demanda física do treinamento e competição é conseguida por meio das adaptações provenientes de agentes estressores aplicados no programa de treinamento.

A pré-temporada é uma fase prévia de fundamental importância dentro do planejamento, pois permite iniciar nos atletas as adaptações funcionais necessárias para suportarem os esforços que a competição vai exigir.

A profundidade e a consistência que as adaptações funcionais provenientes dessa fase de treinamento poderão alcançar são função das capacidades e potencialidades iniciais do atleta, verificadas por meio de testes fisiológicos e laboratoriais, como também das condições de treinamento existentes.

É importante salientar que cada período e etapa do treinamento desenvolvido pelo futebolista devem ser controlados por princípios científicos, bem fundamentados e dentro de um modelo teórico bem definido.

Planejar é, sobretudo, utilizar meios e métodos dinâmicos de organização no processo de treinamento, objetivando condicionar os atletas, ao longo do período competitivo, a um conjunto de aptidões compatíveis com a prática do futebol.

\section{TABELA 4}

Valores médios, mínimos e máximos de potência pico, potência média e do índice de fadiga muscular determinados em teste não invasivo de potência anaeróbia Wingate em jogadores de futebol profissional $(n=23)$ antes do início da pré-temporada

\begin{tabular}{ccc}
\hline $\begin{array}{c}\text { Potência pico } \\
\left(\mathbf{w} \cdot \mathbf{k g}^{-\mathbf{1}} \mathbf{)}\right.\end{array}$ & $\begin{array}{c}\text { Potência média } \\
\left(\mathbf{w . k g} \mathbf{~}^{-1}\right)\end{array}$ & $\begin{array}{c}\text { Índice de fadiga } \\
\mathbf{( \% )}\end{array}$ \\
13,5 & & \\
$\pm 1,1$ & 10,1 & 53,0 \\
$(11,4-15,6)$ & $\pm 0,6$ & $\pm 7,7$ \\
& $(8,8-11,1)$ & $(38-69)$ \\
\hline
\end{tabular}




\section{TABELA 5}

Planejamento e controle fisiológico do treinamento físico dos jogadores de futebol profissional da Associação Portuguesa de Desportos de São Paulo em pré-temporada realizada na Cidade de Águas de Lindóia, interior do Estado

\begin{tabular}{|c|c|c|c|c|c|c|}
\hline & DIA 1 & DIA 2 & DIA 3 & DIA 4 & DIA 5 & DIA 6 \\
\hline \multirow[t]{6}{*}{ SEMANA 1} & $7 / 1$ & $8 / 1$ & $9 / 1$ & $10 / 1$ & $11 / 1$ & $12 / 1$ \\
\hline & Manhã & Manhã & Manhã & Manhã & Manhã & Manhã \\
\hline & $\begin{array}{c}\text { Intervalado aeróbio } \\
8 \text { tiros de } 1.000 \mathrm{~m} \\
\text { lactato } \leq 4 \mathrm{mM} \leq \mathrm{LV} 2 \\
+ \\
\text { alongamento }\end{array}$ & $\begin{array}{c}\text { Circuito c/ obstáculos } \\
+ \\
\text { lactato entre } \\
4 \text { e } 5 \mathrm{mM} \\
+ \\
\text { alongamento }\end{array}$ & $\begin{array}{c}\text { Intervalado aeróbio } \\
8 \text { tiros de } 1.000 \mathrm{~m} \\
\text { Corrida intervalada } \\
\leq \mathrm{LV} 2 \\
+ \\
\text { alongamento }\end{array}$ & $\begin{array}{c}\text { 8km (aeróbio) } \\
\text { lactato } \leq 4 \mathrm{mM} \\
\text { lactato } \leq 4 \mathrm{mM} \leq \mathrm{LV} 2 \\
+ \\
\text { alongamento }\end{array}$ & $\begin{array}{c}\text { Intervalado } \\
\text { lático }(250 \mathrm{~m}) \\
\text { lactato } \geq 10 \mathrm{mM} \\
+ \\
\text { alongamento }\end{array}$ & $\begin{array}{c}\text { Treino técnico } \\
\text { lactato entre } 2 \text { e } \\
4 \mathrm{mM} \\
+ \\
\text { alongamento }\end{array}$ \\
\hline & Tarde & Tarde & Tarde & Tarde & Tarde & Tarde \\
\hline & $\begin{array}{c}\text { musculação a } 60 \% \\
\text { da carga máxima } \\
\text { lactato } \leq 4 \mathrm{mM} \leq \mathrm{LV} 2 \\
\text { alongamento } \\
+ \\
\text { piscina }\end{array}$ & $\begin{array}{c}\text { 8km (aeróbio) } \\
\text { contínuo/lactato } \\
4 \mathrm{mM} \leq \mathrm{LV} 2 \\
+ \\
\text { alongamento } \\
+ \\
\text { piscina }\end{array}$ & $\begin{array}{c}\text { musculação a } 60 \% \\
\text { da carga máxima } \\
\text { alongamento } \\
+ \\
\text { piscina }\end{array}$ & Folga & $\begin{array}{c}\text { Intervalado } \\
\text { aeróbio } \\
\text { lactato } \leq 4 \mathrm{mM} \\
\leq \mathrm{LV} 2 \\
+ \\
\text { alongamento } \\
+ \\
+ \\
\text { piscina }\end{array}$ & $\begin{array}{c}\text { Treino } \\
\text { técnico-tático } \\
\text { coletivo } 10 \\
\text { rec. } 1 \text { a } 2 ' \\
\text { coletivo } 20 \\
\text { s/rec.lactato } \\
\text { entre } 4 \text { e } 6 m M \\
+ \\
\text { alongamento }\end{array}$ \\
\hline & DIA 7 & DIA 8 & DIA 9 & DIA 10 & DIA 11 & DIA 12 \\
\hline \multirow[t]{6}{*}{ SEMANA 2} & $13 / 01$ & $14 / 01$ & $15 / 01$ & $16 / 01$ & $17 / 01$ & $18 / 01$ \\
\hline & Manhã & Manhã & Manhã & Manhã & Manhã & Manhã \\
\hline & $\begin{array}{c}\text { Intervalado } \\
\text { anaeróbio lático } \\
\begin{array}{c}(250 \mathrm{~m}) \\
\geq 10 \mathrm{mM} \\
+\end{array} \\
\text { alongamento }\end{array}$ & $\begin{array}{c}\text { Coletivo } \\
60^{\prime} \\
+ \\
\text { alongamento } \\
\text { piscina }\end{array}$ & Folga & $\begin{array}{c}\text { Coletivo } \\
70^{\prime} \\
+ \\
\text { alongamento } \\
\text { piscina }\end{array}$ & $\begin{array}{c}\text { Coletivo } \\
70^{\prime} \\
+ \\
\text { alongamento } \\
\text { piscina }\end{array}$ & $\begin{array}{c}\text { Treino } \\
\text { técnico } \\
+ \\
\text { alongamento } \\
\text { piscina }\end{array}$ \\
\hline & Tarde & Tarde & Tarde & Tarde & Tarde & Tarde \\
\hline & $\begin{array}{c}\text { aeróbio contínuo } \\
\text { lactato } 2 \text { a } 3 \mathrm{mM} \\
+ \\
\text { treino técnico }\end{array}$ & $\begin{array}{c}\text { Musculação a } \\
60 \% \text { da carga máx. } \\
+ \\
\text { alongamento } \\
\text { piscina }\end{array}$ & $\begin{array}{c}\text { Recreativo } \\
+ \\
\text { alongamento } \\
\text { piscina }\end{array}$ & $\begin{array}{c}\text { Musculação a } \\
60 \% \text { da carga máx. } \\
+ \\
\text { alongamento } \\
\text { piscina }\end{array}$ & Folga & $\begin{array}{l}\text { J ogo } \\
\text { amistoso }\end{array}$ \\
\hline & DIA 13 & DIA 14 & DIA 15 & DIA 16 & DIA 17 & DIA 18 \\
\hline \multirow[t]{5}{*}{ SEMANA 3} & $19 / 01$ & $20 / 01$ & $21 / 01$ & $22 / 01$ & $23 / 01$ & $24 / 01$ \\
\hline & Manhã & Manhã & Manhã & Manhã & Manhã & Manhã \\
\hline & $\begin{array}{c}\text { aeróbio contínuo de } \\
40 \mathrm{~min} \\
\mathrm{LV} 1 \leq \mathrm{LV} 2 \\
+ \\
\text { Piscina } \\
+ \\
\text { alongamento }\end{array}$ & $\begin{array}{c}\text { Treino técnico } \\
2 \leq 4 \mathrm{mM} \\
\text { Anaeróbio alático } \\
\text { velocidade ( } 20 \text { a } 30 \mathrm{~m}) \\
\text { a } 95 \% \text { do máximo } \\
+ \\
\text { alongamento }\end{array}$ & Folga & $\begin{array}{c}\text { Treino técnico } \\
\text { tático } \\
+ \\
\text { alongamento }\end{array}$ & $\begin{array}{c}\text { Treino físico } \\
\text { técnico } \\
+ \\
\text { alongamento }\end{array}$ & $\begin{array}{c}\text { Concentração } \\
\text { no hotel }\end{array}$ \\
\hline & Tarde & Tarde & Tarde & Tarde & Tarde & Tarde \\
\hline & $\begin{array}{l}\text { viagem para } \\
\text { São Paulo }\end{array}$ & $\begin{array}{c}\text { Jogo } \\
\text { coletivo } \\
+ \\
\text { alongamento }\end{array}$ & $\begin{array}{c}\text { Treino técnico } \\
\text { tático + jogo } \\
\text { coletivo } \\
+ \\
\text { alongamento }\end{array}$ & $\begin{array}{c}\text { Treino técnico } \\
\text { Jogo coletivo } \\
+ \\
\text { alongamento }\end{array}$ & $\begin{array}{c}\text { Recreativo } \\
+ \\
\text { Concentração } \\
\text { no hotel }\end{array}$ & $\begin{array}{c}\text { J ogo } \\
\text { oficial } \\
\text { início do } \\
\text { campeonato } \\
\text { paulista }\end{array}$ \\
\hline
\end{tabular}

Fisicultores: Luís Inarra e J osé Roberto Rivelino; Médico: Alberto Azevedo Alves Teixeira; Fisiologista: Paulo Roberto Santos Silva 
TABELA 6

Valores médios, mínimos e máximos dos testes de campo nas distâncias de $100 \mathrm{~m}, 200 \mathrm{~m}, 300 \mathrm{~m}$ e $1.000 \mathrm{~m}$ determinados em jogadores de futebol profissional $(n=23)$ antes do início da pré-temporada

\begin{tabular}{lcccc}
\hline & $\mathbf{1 0 0 m}$ & $\mathbf{2 0 0 m}$ & $\mathbf{3 0 0 m}$ & $\mathbf{1 . 0 0 0 m}$ \\
Tempo & $13^{\prime \prime} 90$ & $29^{\prime \prime} 61$ & & \\
(seg. e min $)$ & \pm 07 & $\pm 1^{\prime \prime} 3$ & $46^{\prime \prime} 50$ & $3^{\prime} 32^{\prime \prime}$ \\
& $\left(12^{\prime \prime} 88-15^{\prime \prime} 00\right)$ & $\left(27^{\prime \prime} 15-31^{\prime \prime} 50\right)$ & $\left(42^{\prime \prime} 58-52^{\prime \prime} 00\right)$ & $\left(3^{\prime} 11^{\prime \prime}-3^{\prime \prime} 50^{\prime \prime}\right)$ \\
\hline
\end{tabular}

TABELA 7

Valores médios, mínimos e máximos da avaliação isocinética computadorizada da articulação dos joelhos a velocidades angulares de $60^{\circ} \mathrm{S}^{-1}$ e $300^{\circ} \mathrm{S}^{-1} \mathrm{em}$ jogadores de futebol profissional $(n=23)$ antes do início da pré-temporada

\begin{tabular}{lccccc}
\hline & \multicolumn{4}{c}{ Velocidades angulares } \\
\cline { 2 - 3 } & \multicolumn{2}{c}{$\mathbf{6 0}^{\circ} \mathbf{S}^{-\mathbf{1}}$} & & \multicolumn{2}{c}{$\mathbf{3 0 0}^{\circ} \mathbf{S}^{-\mathbf{1}}$} \\
\cline { 2 - 3 } \cline { 5 - 6 } & Extensão & Flexão & & Extensão & Flexão \\
& & & & 137 & 121 \\
Perna direita (Nm) & 298 & 198 & & \pm 32 & \pm 26 \\
& $(156-490)$ & $(136-202)$ & & $(96-218)$ & $(69-159)$ \\
\hline Perna esquerda (Nm) & 272 & 185 & & 138 & 122 \\
& \pm 62 & \pm 45 & & \pm 28 & \pm 27 \\
& $(198-454)$ & $(102-286)$ & & $(104-210)$ & $(81-160)$ \\
\hline
\end{tabular}

Portanto, dentro do processo global de treinamento, tornase necessário preocupar-se com o controle de três fases que regem a forma físico-desportiva de um atleta: $1^{\underline{a}}$ ) aquisição da forma (fase preparatória); 2aㅡ) manutenção (fase competitiva); e 3a) "perda" (fase de transição). Contudo, a fase transitória para uma equipe de futebol no Brasil tem papel importante. Pois, ao início da pré-temporada, os atletas que menos perderam condicionamento físico motivado por algum tipo de treinamento realizado nesse período, conseqüentemente, serão aqueles que menos terão diminuição das adaptações funcionais decorrentes de toda a carga anual de treinamento recebida, não sendo portanto afetados pela síndrome de abstinência do exercício. Logo, esses se readaptarão mais rapidamente aos treinamentos realizados na fase preparatória, o que constitui uma vantagem.

Portanto, a verificação do estágio inicial de condição física dos futebolistas só é possível após uma avaliação funcional multidisciplinar.

É importante salientar que a organização do período transitório no futebol, ou seja, o período entre a última competição, as férias e início do ano futebolístico, merece atenção especial.

Em geral, existem duas possibilidades de organização do período de transição para os jogadores. A primeira é o repou- so passivo, o futebolista não realiza qualquer tipo de atividade sistematizada. A segunda - e a mais adequada para o futebolista, em nossa opinião - é a recuperação ativa, por meio da implantação de um programa de treinamento, com determinadas características, sem as formalidades exigidas durante o ano competitivo.

Não nos podemos esquecer que o calendário do futebol brasileiro é uma situação em que a organização da modalidade impõe aos especialistas um curto período preparatório e um longo período competitivo.

Portanto, quando iniciamos uma pré-temporada (período preparatório), a filosofia de introdução das cargas de treinamento é baseada, num primeiro momento, em seu aumento de volume e, posteriormente, durante a competição, em intensidade (ajuste fino), utilizando-se, até certo ponto, o princípio da progressão.

Entretanto, é importante salientar que, dependendo do resultado dos testes fisiológicos, já na pré-temporada, é possível adiantar fases de treinamento que seriam desenvolvidas somente após a competição ter sido iniciada. Essa é uma das vantagens que se apresenta com a avaliação funcional prévia do futebolista.

Contudo, é importante salientar que os jogadores que voltam do período de férias em boas condições físicas facilitam o 
trabalho da comissão técnica, que pode direcionar o treinamento específico com antecedência.

Entretanto, como é sabido, o tempo disponível para atingir o melhor da forma atlética do futebolista é escasso. Assim, em meio da fase competitiva, ainda se procura desenvolver o nível ideal de condicionamento físico para jogar futebol.

A chamada fase de manutenção da carga é aplicada, após alguns meses, e conseguida com a divisão entremeada dos treinamentos específicos de ordem física, técnica e tática.

No presente estudo, após a realização dos testes, no laboratório de fisiologia do exercício e de campo, os futebolistas foram divididos em grupos e, de acordo com os índices atingidos, foi estabelecida a carga de treinamento adequada a cada atleta, em função do objetivo pretendido. Foi respeitado o verdadeiro princípio da individualidade funcional de cada futebolista.

Os treinamentos foram desenvolvidos em dois períodos (manhã e tarde) [tabela 5]. Na primeira semana, houve distribuição de um volume maior de treinamento, direcionado para o aprimoramento da capacidade cardiorrespiratória e muscular, com a utilização de exercícios aeróbios contínuos e intervalados, exercícios de flexibilidade complementados com musculação e piscina. A partir da segunda semana, o treinamento foi mesclado, com exercícios aeróbios e anaeróbios. O controle fisiológico da carga de treinamento foi realizado por meio de medidas individuais de lactato sanguíneo (tabela 5).

Na terceira e última semana de preparação, foram intensificados os treinamentos com bola, em que os futebolistas realizaram exercícios técnicos, táticos e jogo coletivo.

A estratégia utilizada pela comissão técnica foi monitorar a intensidade de todos os treinamentos, por meio de medidas de lactato em campo, inclusive durante os jogos coletivos, como meio de estimar a sobrecarga funcional dos futebolistas. É importante salientar que, apesar de toda a tecnologia utilizada no controle de treinamento dos futebolistas, o tempo disponível para o desenvolvimento das valências físicas para jogar futebol de competição foi curto. Em nosso caso, a pré-temporada teve duração de apenas 16 dias (tabela 5).

Portanto, o controle fisiológico das cargas de treinamentos provenientes da avaliação funcional é uma medida que deve ser valorizada, pois as informações obtidas podem determinar treinamentos objetivos para o aumento da aptidão física do futebolista.

Concluindo, de nosso ponto de vista, esse é o verdadeiro sentido de submeter jogadores de futebol, amadores ou profissionais, à avaliação funcional multidisciplinar, como procedimento inicial para o controle fisiológico do treinamento físico realizado numa pré-temporada futebolística. É importante salientar que a infra-estrutura de apoio ao futebolista deve ser valorizada pelos dirigentes, pois, sobretudo, a integridade física do jogador de futebol, como patrimônio do clube, está em jogo e deve ser preservada a todo custo. Além disso, a descrição dos procedimentos desse trabalho não teve a intenção de impor aos colegas um modelo obrigatório a ser seguido, mas alertar sobre a importância de uma avaliação funcional prévia como meio de planejar objetivamente o programa de treinamento do futebolista.

É importante lembrar que a contínua e sistemática ligação entre a teoria e a prática, como também saber analisar criticamente o conteúdo da prática desenvolvida e de ser capaz de operacionalizar os conceitos teóricos, são condição fundamental para fazer evoluir o processo de treinamento em qualquer modalidade esportiva.

\section{AGRADECIMENTOS}

Agradecemos à professora Angela Romano, do Instituto do Coração do Hospital das Clínicas da Faculdade de Medicina da USP, por sua colaboração na execução deste manuscrito, e ao professor de língua inglesa Humberto Blancato pela tradução do abstract.

\section{REFERÊNCIAS}

1. Astrand PO, Rodahl K. Textbook of work physiology. New York: McGraw-Hill, 1977.

2. Silva PRS, Visconti AM, Teixeira AAA, Romano A, et al. Avaliação funcional multivariada em jogadores de futebol profissional - Uma metanálise. Rev Bras Med Esporte 1998;4:182-96.

3. Yazbek Jr P, Battistella LR. Condicionamento físico do atleta ao transplantado. Aspectos multidisciplinares na prevenção e reabilitação cardíaca. 1a ed. Editora Sarvier/APM, 1994.

4. Silva PRS, Romano A, Yazbek Jr P, Battistella LR. Ergoespirometria computadorizada ou calorimetria indireta: um método não invasivo de crescente valorização na avaliação cardiorrespiratória ao exercício. Rev Bras Med Esporte 1998;4:147-58.

5. Borg G. Perceived exertion as an indicator of somatic stress. Scan J Rehab Med 1970;2:92-6.

6. Noble BJ. Clinical applications of perceived exertion. Med Sci Sports Exerc 1982;14:406-11.

7. Bhambhani Y, Singh M. Ventilatory thresholds during a graded exercise test. Respiration 1985;47:120-8.

8. Bar-Or O. The Wingate anaerobic test: an update on methodology, reliability and validity. Sports Med 1987;4:381-94.

9. Faulkner JA. Physiology of swimming and diving. In: Falls H. Exercise physiology. Baltimore: Academic Press, 1968.

10. Glantz SA. Primer of biostatistics. $3^{\text {rd }}$ ed. New York: MacMillan Publishing, 1978. 